\title{
Sidelight on the Diffuse Interstellar Bands Problem
}

\author{
Frédéric Zagury*
}

Institut Louis de Broglie, 23 rue Marsoulan, 75012 Paris, France

\begin{abstract}
I investigate the conditions upon which atmospheric absorption may participate to the observation of a diffuse interstellar band (DIB), and the implications it would have. A necessary condition is that the spectrum of reddened stars comprises a few percent of starlight forward scattered by the interstellar cloud on the line of sight. Reciprocally, this scattered starlight could, in part, explain the complexity of the DIB spectrum and several observed DIB properties. It will also affect the interstellar extinction curve and the value of the $R_{V}$ parameter.
\end{abstract}

Keywords: Atmospheric effects, ISM: dust, extinction, ISM: lines and bands.

\section{INTRODUCTION}

Diffuse interstellar bands (DIBs) represent a longstanding spectroscopic problem in astronomy, which appeared, nearly concomitant to the discovery of the interstellar medium, in the 1920-30 ([1,2], see also the introduction in [3]).

DIBs are generally weak (a few percent of the continuum) absorption features clearly observed in the visible spectrum of most reddened stars, under circumstances which are not understood. They seem to be absent from the spectrum of unreddened stars, and their strength roughly correlates with the amount of color excess, $E(B-V)=A_{B}-$ $A_{V}$, along the line of sight. They are thus logically attributed to absorption in interstellar clouds.

DIB strength's dependence on reddening does not follow a linear or simple law. Some DIBs are observed towards very low column density directions $(E(B-V) \ll 0.1,[4,5])$. There is an average linear rise of DIB strength at moderate $E(B-V)$, but DIBs tend to be deficient at high reddenings [6-8]. It has therefore been suggested that DIB absorption arises from the outer, low density parts of interstellar clouds. DIBs are generally much weaker in the spectra of stars illuminating a nebula or with circumstellar dust $([8,9]$, and Sect. 4.1).

Merrill [10], and Duke [11] have noted an increase of DIB strength with stellar distance. This somewhat anachronistic dependence is reported by Herbig [8] but has not been explained or re-investigated in recent studies.

DIB detections have increased exponentially during the last decades (half a dozen in the 1940, 40 in the mid-1970s, a hundred in 1991, over 380 in the recent Hobbs et al. [12] survey), along with instrumental progress.

There is, to this date, no identification of DIB carriers (see [8] for a review). There seems to be a consensus on a molecular origin, although the dependence of DIB strength on atomic hydrogen column density, $N(\mathrm{HI})$, rather than on $N\left(\mathrm{H}_{2}\right)$,

*Address for correspondence to this author at the Institut Louis de Broglie, 23 rue Marsoulan, 75012 Paris, France; E-mail: fzagury@wanadoo.fr the column density of molecular hydrogen [13], and Meyer's [4] calculations (which indicate that molecules should not be abundant enough to produce DIBs in low column density regions), hamper the hypothesis. The idea that molecules could be synthesized in dense clouds before being released, and eventually transformed, in the diffuse interstellar medium seems to me a bit far fetched (how do the DIB carriers reach the diffuse high latitude interstellar medium?). PAHs (polycyclic aromatic hydrocarbon mole-cules) are often cited as DIB carriers, but no evidence supports the claim $[8,14]$. There is anyway no published spectrum of PAHs or any other supposed interstellar material that would reproduce even part of the DIB spectrum.

In general, reviews on DIB properties leave an impression of complexity one would a-priori not expect in a basic, close to vacuum, interstellar cloud, furthermore in its lowest density parts. It may also seem surprising that a medium consisting of a few atoms and molecules per $\mathrm{cm}^{3}$ can give rise to a chemistry we are not able to reproduce on earth.

My interest for the DIBs was motivated by earlier studies on the Red Rectangle nebula. The spectrum of the nebula exhibits the broad ERE (Extended Red Emission) bump in the red, and emission-like bands, which, as the DIBs, remain unidentified [15-17]. Some of these bands have been considered as DIBs in emission [15, 17-19].

A few Red Rectangle bands, some of which are associated to DIBs, are found in the background spectra of the observations $[20,21]$. This is particularly obvious in the $6200 \AA$ A wavelength region, and I have shown the remarkable similarity which exists between the spectra of DIB complex $\lambda 6200$ and atmospheric $\mathrm{NO}_{2}$ (Fig. 5 and sect. 3.3 in [21]).

These observations may suggest that some DIBs are due to absorption in the atmosphere, under conditions which need to be understood, since, as mentioned above, DIB observations require to have an interstellar cloud on the line of sight.

It is this hypothesis I would like to further in this paper. In Sect. 2, I investigate the conditions for a DIB carrier to belong to the atmosphere, and in Sect. 4 several implications 
are analyzed and compared to observation. In-between, Sect. 3 is an attempt to relate these conditions to a remark by G. Herbig [8].

\section{CONDITIONS FOR A ROLE OF THE ATMOSPHERE IN DIB FORMATION}

\subsection{Can a DIB Arise from Absorption in the Earth's Atmosphere?}

Obviously, a DIB carrier must belong to one of the two media superimposed along the sight-line of a reddened star, an interstellar cloud and the atmosphere. The interstellar cloud further seems to be necessary to the observation of the DIB.

If starlight at a specific DIB wavelength is absorbed in the atmosphere (and not in the interstellar cloud), the only way for the interstellar cloud to contribute to the spectrum of the star is through a component of scattered light.

If the DIB carrier belongs to the atmosphere, direct light from the star shouldn't be more affected by atmospheric absorption than light from an unreddened star (the atmosphere has no way to distinguish one from the other).

It follows that observation of a DIB which is due to absorption in the atmosphere requires that the spectrum of a reddened star contains a proportion of scattered light by the interstellar cloud on the line of sight, and that the DIB results from selective absorption in the atmosphere of this scattered light component.

This latter condition is in fact very similar to conclusions I have reached from the analysis of observations presented in previous papers $[20,22,23]$. These observations indicate that atmospheric absorption is, for reasons I have not been able to determine (atmospheric turbulence first comes to mind, although I would not exclude an instrumental bias), more efficient on faint extended objects (nebulae, galaxies) than on stars. Decomposition of the spectrum of a reddened star into direct and scattered starlight corresponds to the configuration where the observer observes simultaneously a star and its nebula.

\subsection{Orders of Magnitude}

If a DIB arises due to absorption in the atmosphere, the scattered starlight component in the spectrum of a reddened star must represent a few percent of the continuum.

Scattering by an interstellar cloud can be of two types: forward-directed scattering by large interstellar grains, or isotropic Rayleigh scattering by atoms, molecules, or tiny dust grains.

The single scattering model used in [24] with an albedo of $\sim 0.7$ shows that up to $20 \%$ of the incident flux of the star at the cloud location can be scattered by large interstellar grains over all directions. For a strong forward-scattering phase function, known to be the common case in the interstellar medium [25], most of this light is thrown in a small angular angle in the forward direction, and may thus represent a few percent of the starlight received on earth.
Rayleigh scattering is far less efficient than forward scattering and will in general require, to be perceived, the source of light to be close to the cloud. Even so it remains weak: if, for instance, light from the Red Rectangle nebula is, in the red, Rayleigh scattering by hydrogen [23], it does not exceed $1 \%$ of the light we receive from HD44179's direction.

Scattering (isotropic or forward scattering) can be greatly enhanced if the scattering medium exhibits some kind of an order, for example because of turbulence (see [26] for enhanced scattering in turbulent media). I have argued [27], several years ago, that an addition of coherent scattered starlight in the spectrum of reddened stars would explain the departure from linearity of interstellar extinction in the UV. The scattered component necessary to explain the atmospheric origin of a DIB could be the visible tail of this coherent scattered light.

There are thus various possibilities which can explain a few percent component of scattered starlight in the spectrum of reddened stars, and the observed strength of the DIBs.

\section{RELATION TO PREVIOUS WORKS}

Several DIB properties, as the dependence of DIB strength on reddening (see the introduction and Sect. 4.2), are reminiscent of scattering processes. This may have influenced the following comment by G. Herbig on the absence of DIBs in circumstellar matter: 'A more prosaic explanation is, however, also possible: If the diffuse interstellar bands are formed by a pure scattering process and the dust is spherically distributed around the central source, then the bands would disappear in integrated light.' (sect. 2.4, p.29 in [8]).

The sentence stands curiously against the rest of G. Herbig's paper. The idea might appear confuse to the reader who may wonder how a DIB can be formed through a pure scattering process, and why should the band disappear in integrated light from circumstellar dust? This is unfortunately nowhere justified or deepened, in [8] or in any other of G. Herbig's articles.

I do not see how DIBs can be pure scattering, unless to accept, as in Sect. 2.1, a component of scattered starlight in the spectrum of reddened stars. Then, features in the scattering cross-section, or absorption bands of the scatterers could provoke the DIBs. This is as if atmospheric absorption, as it has been considered in the previous section, was replaced by an absence of scattering at DIB wavelengths. Orders of magnitude needed for the scattered light will be as found in the preceding section, and consequences (next section) will be the same.

\section{IMPLICATIONS}

In this section, I suppose that a DIB, in either case of Sects. 2 and 3, is due to the presence of a few percent of scattered starlight in the spectrum of a reddened star. I investigate how different parameters, the geometry and the reddening, can influence the amount of scattered starlight (thus the DIB's strength), and the effect it will have on the visible extinction curve. 


\subsection{Influence of the Geometry}

In the two cases which may justify a few percent of scattered starlight in the spectrum of reddened stars, forward scattering by large grains or coherent scattering, angles of scattering need to be small (say within a maximum angle $\left.\theta_{\max }\right)$. The amount of scattered starlight will thus depend on the geometry of the scattering.

The area of the interstellar cloud which contributes most to the scattering, that is the area around the direction of the star within which the scattering angle is less than $\theta_{\max }$, grows with distance between the star and the cloud. It is maximum when the star is at infinity (the distance between the star and the cloud is much larger than the sun to cloud distance), minimum when the cloud is in the vicinity of the star.

Strength of a DIB linked to scattered starlight should thus have a general tendency to increase with star distance. If the DIB is observed in the spectrum of the illuminating star of a nearby (close to the star) foreground cloud, it should be weaker than if the star was farther away in the clear. These two tendencies are conform to observation [3, 10, 11].

The DIB should be particularly weak in stars embedded in circumstellar matter. HD44179, the star at the center of the Red Rectangle, surrounded by a very thick torus of matter [28, 29], has no DIB in its spectrum and may be an example.

DIBs in circumstellar matter have been observed and discussed in several papers ([8,9] and references therein). There is, in general, no significant DIB detection, except in two studies [30, 31].

Le Bertre \& Lequeux [30] detects DIB $\lambda 6177$ in the direction of several stars with circumstellar dust. This DIB is particularly interesting since it is a clear feature in the spectrum of $\mathrm{NO}_{2}[21]$.

Le Bertre and Lequeux assume that stars in their sample are not affected by foreground reddening. This is far from being obvious and remains to be proved: in all of the directions where the authors detect $\lambda 6177$ the 'large scale' reddening (due to cirrus on the line of sight) given by NED database ([32], http://nedwww.ipac.caltech.edu/forms/calculator. html) is particularly important, sometimes even larger than the reddening of the stars. In contrast, in the directions where NED's $E(B-V)$ is low or less than the stars' reddening, Le Bertre and Lequeux do not observe the DIB. It therefore seems to me probable that $\lambda 6177$, when it is detected in these observations, is due to foreground interstellar clouds (and not to circumstellar dust, in agreement with all observations of stars reddened only by circumstellar material).

In the direction of supergiant O9.5 HD188209 Galazutdinov et al. [31] report days variations of some DIBs. The authors rightly argue that such a variability would not be observed if the DIB carrier(s) belonged to an intervening interstellar cloud. It thus has to arise either in the immediate vicinity of the star (as supposed in [31]), or in the atmosphere.

The reddening of HD188209 $[E(B-V)=0.20$, from $b-$ $v \sim-0.07$ and $\left.(B-V)_{0} \sim-0.27\right]$ is the same as the one expected from the galactic cirrus on the line of sight $(E(B-V)=$ 0.20 , from NED). The star is also relatively far-away from the Sun (Hipparcos gives a parallax of 0.22 mas), and has a bump at $2200 \AA$, usually absent in the observation of stars with circumstellar dust. HD188209 is furthermore not visible in IRAS 60 and $100 \mathrm{~mm}$ images, while heated dust in its vicinity should give a bright spot at the position of the star on the infrared maps. These observations favor a position of HD188209 behind the interstellar cloud on the line of sight, which would imply that the day variations observed in the spectrum of HD188209 are not due to circumstellar matter but to the earth's atmosphere.

\subsection{Variation of DIB Strength with Reddening}

In a given geometry, the amount of scattered light, and the DIB's strength, will increase linearly with reddening at small optical depth and reach a ceiling when the optical depth is close to 1 .

For larger optical depths, scattered starlight diminishes because of multiple scattering and absorption. It might explain that DIBs are more difficultly observed in high column density directions $[6,7]$.

In these directions it is however difficult to predict what will be the proportion of scattered light in the observed spectrum of the star, since direct starlight is also extinguished. For high optical depths scattered light should nevertheless decrease more quickly than direct starlight.

\subsection{Effects on the Extinction Curve}

A scattered starlight component in the spectrum of reddened stars will basically be a continuum, distributed over the whole spectrum. Observed extinction curves, which include this component, might thus be different from the true interstellar extinction.

The true visible extinction curve will best be determined from observations of stars with very low reddenings (scattered starlight is small). In these directions, the extinction curve is linear in $1 / \lambda$ over the whole visible-UV wavelength range, in the Milky Way as well as in the Magellanic Clouds [33, 34]. Therefore, if a component of scattered starlight does modify the true extinction curve (and provided that there is no grey extinction, see eq. 2 in [34]), the true value of $R_{V}$ should be 4 .

In addition, for observations from the ground, the continuum of scattered starlight will be affected by atmospheric absorption at all wavelengths. This absorption will reflect the complexity of atmospheric absorption, so that a high number of DIBs should be found with increasing resolution and signal-to-noise ratio.

\section{CONCLUSION}

In this study I have first searched for the conditions upon which a DIB can arise due to the atmosphere, and the implications it has. I finally found that a component, in the spectrum of reddened stars, of starlight scattered by the interstellar cloud on the line of sight could explain a large set of the observed properties of the DIBs. A DIB related to this component may then be atmospheric (absorption by 
molecules of the atmosphere) or interstellar (interruption of the scattered light at the DIB wavelength).

Conditions for a DIB to arise due to the earth's atmosphere are that the spectrum of a reddened star contains a few percent of scattered starlight by the interstellar cloud on the line of sight, and that this light be more specifically absorbed, at the DIB wavelength, by the atmosphere. If one DIB only is due to absorption in the atmosphere (for instance 26177), many others can be expected, reflecting the richness and complexity of atmospheric absorption. A role of the atmosphere in DIB observations, surprising at first glance, meets earlier results I have obtained from the analysis of nebular and galaxies' spectra, especially in the Red Rectangle.

The article largely focused on the implications a component of scattered starlight in the spectrum of reddened stars would have on observation. A key parameter is the relative distance between the interstellar cloud, the reddened star, and the observer: DIBs related to the scattered light should be absent in the spectrum of stars with circumstellar matter; the expected dependence of DIB strength on stars' distances and on reddening agrees with the observations.

A component of scattered light in the spectrum of reddened stars will also have consequences for the visible extinction curve. It may imply that true extinction in the visible is simply linear, due to a size-distribution of large grains. In this case, the exact value of $R_{V}$ must be 4 .

Some DIBs have been observed in other galaxies [35, 36], with a significant redshift, and $\lambda 4430$ seems to be present in a few Hubble Space Telescope (HST) observations [37]. These observations question, but do not necessarily diminish the interest of the present work. There are first very few such DIBs, and they could be related to the scattered starlight as indicated in Sect. 3.

It is also noteworthy that, besides $\lambda 4430$, no DIB has yet been observed from space. Observations with the International Ultra-violet Explorer (IUE) prove that there is no DIB under $3000 \AA[38,39]$. In the visible, stars in LMC's RMC136a association, observed by the HST with a possible absorption feature at $4430 \AA$ [37], do not show DIBs at longer wavelengths. This may be attributed to an insufficient signal-to-noise ratio, but would worth new observations with the HST.

This study finally shows that other solutions than a pure interstellar absorption process may be envisaged for the DIB problem. The hypothesis at the origin of this research, a role of the atmosphere in the observation of some DIBs, may be wrong, or only partially right. It has the advantage to account for several DIB observations, for the resemblance between the DIB spectrum and the spectrum of $\mathrm{NO}_{2}$ in the $6200 \AA$ region, and may explain part of the DIB spectrum complexity. This should, in my opinion, motivate and justify new observations from space.

\section{REFERENCES}

[1] Heger ML. Further study of the sodium lines in class B stars: The spectra of certain class B stars in the regions 5630A-6680A and 3280A-3380A; Note on the spectrum of $\gamma$-Cassiopeiae between $5860 \AA ̊$ And 6600 Å. Lick Obs Bull 1922; 10: 141-8.
[2] Merrill PW. Unidentified Interstellar Lines. Publ Astron Soc Pac 1934; 46: 206-7.

[3] Herbig GH. The diffuse interstellar bands : IV - The region 44006850 A. Astrophys J 1975; 196: 129-60.

[4] Meyer DM. Observations of diffuse interstellar lines toward stars with low column densities of $\mathrm{H}_{2}$. Astrophys J 1983; 266: L51-4.

[5] Galazutdinov GA, Krelowski J, Moutou C, Musaev FA. Diffuse interstellar bands in low E(B-V) objects. Mon Not R Astron Soc 1998; 295: 437-42.

[6] Snow TP, Cohen JG. Diffuse interstellar band formation in dense clouds. Astrophys J 1974; 194: 313-22.

[7] Adamson AJ, Whittet DCD, Duley WW. Diffuse interstellar bands in the Taurus dark clouds. Mon Not R Astron Soc 1991; 252: 23445.

[8] Herbig GH. The diffuse interstellar bands. Annu Rev Astron Astrophys 1995; 33: 19-74.

[9] Luna R, Cox NL, Satorre MA, Garcia Hernández DA, Suárez O, Garcia Lario P. A search for diffuse bands in the circumstellar envelopes of post-AGB stars. Astron Astrophys 2008; 480: 133-48.

[10] Merrill PW. Tests of the lines $\lambda 5780$ and $\lambda 6284$ in stellar spectra. Publ Astron Soc Pac 1936; 48: 179-80.

[11] Duke D. Intensities of the interstellar band at $\lambda 4430$. Astrophys J 1951; 113: 100.

[12] Hobbs LM, York DG, Snow TP, et al. A catalog of diffuse interstellar bands in the spectrum of HD204827. Astrophys J 2008; 680: $1256-70$.

[13] Herbig GH. The diffuse interstellar bands : IX - constraints on the identification. Astrophys J 1993; 407: 142-56.

[14] Tielens AGGM. Interstellar polycyclic aromatic hydrocarbon molecules. Annu Rev Astron Astrophys 2008; 46: 289-337.

[15] Van Winckel H, Cohen M, Gull TR. The ERE of the "Red Rectangle" revisited. Astron Astrophys 2002; 390: 147-54.

[16] Schmidt GD, Cohen M, Margon B. Discovery of optical molecular emission from the bipolar nebula surrounding HD 44179. Astrophys J 1980; 239: L133-8.

[17] Fossey SJ. Thesis, University of London, 1990.

[18] Sarre PJ. Diffuse bands in emission. Nature 1991; 351: 356

[19] Scarrott SM, Watkin S, Miles JR, Sarre PJ. Evidence for a link between the more prominent optical emission bands in the Red Rectangle and some of the diffuse instellar absorption. Mon Not R Astron Soc 1992; 255: 11-6.

[20] Zagury F. Analysis of the Schmidt, Cohen \& Margon (1980) features in the Red Rectangle nebula. Open Astron J 2009; 2: 2638.

[21] Zagury F. On the relationship between Red Rectangle and diffuse interstellar band. Open Astron J 2009; 2: 48-57.

[22] Zagury F. Spectral analysis of the background in ground-based long-slit spectroscopy. J Astrophys Astron 2005; 26: 377-94.

[23] Zagury F. An analysis of spectra in the red rectangle nebula. J Astrophys Astron 2005; 26: 395-420.

[24] Zagury F, Boulanger F, Banchet V. Optical images of MCLD123.5+24.9: a cloud illuminated by the North star? Astron Astrophys 1999; 352: 645-58.

[25] Henyey LC, Greenstein JL. Diffuse radiation in the galaxy. Astrophys J 1941; 93: 70-83.

[26] Kharchenko AV, Grésillon D. Visible enhanced scattering from air flow turbulence. Europhys lett 2001; 55: 486-91.

[27] Zagury F. Scattered starlight contamination in the spectrum of reddened stars. New Astron 2001; 6: 415-23.

[28] Roddier F, Roddier C, Graves JE, Northcott MJ. Adaptive optics imaging of proto-planetary nebulae: frosty Leo and the Red Rectangle. Astrophys J 1995; 443: 249-60.

[29] Men'shchikov AB, Balega YY, Osterbart R, Weigelt G. Highresolution bispectrum speckle interferometry and two-dimensional radiative transfer modeling of the Red Rectangle. New Astron 1998; 3: 601-17.

[30] Le Bertre T, Lequeux J. Diffuse absorption bands in the spectra of mass losing objects. Astron Astrophys 1993; 274: 909-16.

[31] Galazutdinov GA, Krelowski J, Musaev FA, Galeev AI. Variability of diffuse interstellar bands in the spectrum of HD 188209. Astron Lett 1999; 25: 656-61.

[32] Schlegel D, Finkbeiner D, Davis M. Maps of dust infrared emission for use in estimation of reddening and cosmic microwave background radiation foregrounds. Astrophys J 1998; 500: 525-53.

[33] Zagury F. The standard theory of extinction and the spectrum of stars with very little reddening. New Astron 2001; 6: 471-86. 
[34] Zagury F. Reddening law and interstellar dust properties along Magellanic sight-lines. Astrophys Space Sci 2007; 312: 113-25.

[35] Ehrenfreund P, Cami J, Jiménez-Vicente J, et al. Detection of diffuse interstellar bands in the magellanic clouds. Astrophys $\mathbf{J}$ 2002; 576: L117-20.

[36] Sollerman J, Cox N, Jakobson P, et al. Diffuse interstellar bands in NGC 1448. Astron Astrophys 2005; 429: 559-67.

[37] Massey P, Bresolin F, Kudritzki RP, Puls J, Pauldrach AWA. The physical properties and effective temperature scale of O-type stars as a function of metallicity. I: a sample of 20 stars in the magellanic clouds. Astrophys J 2004; 608: 1001-27.

[38] Massa D, Savage BD. Measurements of interstellar extinction. In Proceedings of the $135^{\text {th }}$ Symposium of the International Astronomical Union 1989, IAU Symp.; July 1988; Kluwer Academic Publishers, Dordrecht, 1989.

[39] Massa D, Savage BD, Fitzpatrick EL. Peculiar ultraviolet interstellar extinction. Astrophys J 1983; 266: 662-83.

(C) Frédéric Zagury; Licensee Bentham Open.

This is an open access article licensed under the terms of the Creative Commons Attribution Non-Commercial License (http://creativecommons.org/licenses/bync/3.0/), which permits unrestricted, non-commercial use, distribution and reproduction in any medium, provided the work is properly cited. 